\title{
Significance of Parkinson Family Genes in the Prognosis and Treatment Outcome Prediction for Lung Adenocarcinoma
}

\section{OPEN ACCESS}

Edited by:

Florence Le Calvez-Kelm,

International Agency For Research On

Cancer (IARC), France

Reviewed by:

Jasim Khan,

University of Alabama at Birmingham,

United States

Abhishek Jauhari,

University of Pittsburgh, United States

*Correspondence:

Hong Zheng

ziecoe@tmmu.edu.cn

Jigang Dai

daijigang@tmmu.edu.cn

Specialty section:

This article was submitted to

Molecular Diagnostics and

Therapeutics,

a section of the journal

Frontiers in Molecular Biosciences

Received: 02 July 2021 Accepted: 07 September 2021 Published: 20 September 2021

Citation:

Li Y, Lu X, Zhang J, Liu Q, Zhou D, Deng X, Qiu Y, Chen Q, Li M, Yang G,

Zheng H and Dai J (2021) Significance of Parkinson Family Genes in the

Prognosis and Treatment Outcome

Prediction for Lung Adenocarcinoma.

Front. Mol. Biosci. 8:735263.

doi: 10.3389/fmolb.2021.735263

\begin{abstract}
Yanqi $L i^{1}$, Xiao $L u^{1}$, Jiao Zhang ${ }^{1}$, Quanxing Liu ${ }^{1}$, Dong Zhou ${ }^{1}$, Xufeng Deng ${ }^{1}$, Yuan Qiu ${ }^{2}$, Qian Chen ${ }^{3}$, Manyuan $\mathrm{Li}^{1}$, Guixue Yang ${ }^{1}$, Hong Zheng ${ }^{1 *}$ and Jigang Dai ${ }^{1 *}$

${ }^{1}$ Department of Thoracic Surgery, Xinqiao Hospital, Army Medical University (Third Military Medical University), Chongqing, China, ${ }^{2}$ Department of General Surgery, Xinqiao Hospital, Army Medical University (Third Military Medical University), Chongqing, China, ${ }^{3}$ Cancer Center of Daping Hospital, Third Military Medical University (Army Medical University), Chongqing, China
\end{abstract}

Epidemiological investigations have shown that patients with Parkinson's disease (PD) have a lower probability of developing lung cancer. Subsequent research revealed that PD and lung cancer share specific genetic alterations. Therefore, the utilisation of PD biomarkers and therapeutic targets may improve lung adenocarcinoma (LUAD) diagnosis and treatment. We aimed to identify a gene-based signature from 25 Parkinson family genes for LUAD prognosis and treatment choice. We analysed Parkinson family gene expression and protein levels in LUAD, utilising multiple databases. Least absolute shrinkage and selection operator (LASSO) regression was used to construct a prognostic model based on the TCGA-LUAD cohort. We validated the model in external GEO cohorts. Immune cell infiltration was compared between risk groups, and GEO data was used to explore the model's predictive ability for LUAD treatment response. Nearly all Parkinson family genes exhibited significant differential expression between LUAD and normal tissues. LASSO regression confirmed that our seven Parkinson family gene-based signature had excellent prognostic performance for LUAD, as validated in three GEO cohorts. The high-risk group was clearly associated with low tumour immune cell infiltration, suggesting that immunotherapy may not be an optimal treatment choice. This is the first Parkinson family gene-based model for the prediction of LUAD prognosis and treatment outcome. The association of these genes with poor prognosis and low immune infiltration requires further investigation.

Keywords: Parkinson gene family, LUAD, prognosis, tumour mutation burden, neoantigen, immunotherapy

\section{INTRODUCTION}

Parkinson's disease (PD) is the most common neurodegenerative motor disorder. It develops as a result of the premature death of dopamine-containing neurons in a part of the midbrain called the substantia nigra. This leads to a loss of dopaminergic neurons within the substantia nigra pars compacta, depletion of dopamine in the striatum, and the presence of Lewy bodies (Jankovic, 2008). In contrast to the excessive neuronal cell death observed in $\mathrm{PD}$, cancer develops from unrestricted cell proliferation and resistance to cell death (Filippou and Outeiro, 2021). Interestingly, with the development of a more comprehensive understanding of both diseases, an intimate link between PD 
and lung cancer has been gradually revealed. Most epidemiological studies and meta-analyses have reported a lower incidence of lung cancer in $\mathrm{PD}$ patients compared to that in the general population (Catalá-López et al., 2014; Ong et al., 2014; Peretz et al., 2016), highlighting a significant overlap between genes upregulated in PD and downregulated in lung cancer or vice versa (Ibáñez et al., 2014). The intriguing overlap of genes implicated in these two completely different diseases suggests that studying these genes may help improve lung cancer diagnosis, prognosis, and treatment.

Although there is a limited number of studies on Parkinson family genes in cancer, insightful findings have been reported in recent years. Mitophagy, a selective form of autophagy, is the major pathway for the degradation of dysfunctional or superfluous mitochondria in eukaryotic cells (Georgakopoulos et al., 2017), playing a central role in mitochondrial quality control and protection against damaged mitochondria (Tatsuta and Langer, 2008; Youle and Narendra, 2011). The Parkinson family genes PARK2 (PRKN) and PARK6 (PINK1) are considered the main regulators of mitophagy (Yan et al., 2020; Xie et al., 2021). The dysregulation of PRKN- and PINK1mediated mitophagy has thus been suggested as one of the possible mechanisms underlying the pathogenesis of PD (Lin and Beal, 2006). This notion has been preliminarily validated in mouse models (Lu et al., 2014). Interestingly, mitophagy also has a significant impact on the occurrence and development of tumours. A recent study of hepatocellular carcinoma (HCC) suggested that mitophagy triggered by the accumulation of PINK1 and PRKN translocation can promote the apoptosis of HCC cells, suppressing the growth of patient-derived tumour xenografts (Chen et al., 2019). Further, mitophagy can inhibit the growth of pancreatic tumours by attenuating mitochondrial iron accumulation, inflammasome activation, and other processes, with PINK1 and PRKN depletion confirmed to promote KRAS-driven pancreatic tumourigenesis in mouse models (Kang et al., 2019). PARK18 (EIF4G1), an important part of the EIF4F complex, is required for cap-dependent mRNA translation (Jaiswal et al., 2018). Inevitably, EIF4G1 is involved in various cancer-related processes, such as the activation of the mTOR signalling pathway and hypoxia-inducible factor-1a (HIF1a)-related processes (Glück et al., 2018; Lu et al., 2021). Other family members such as PARK22 (CHCHD2), which plays an important role in the switch between catabolism and anabolism (Zacksenhaus et al., 2017), PARK7 (DJ-1), which is involved in ferroptosis (Cao et al., 2020), ubiquitination-related regulatory genes PARK15 (FBXO7) and PARK5 (UCHL1) (Goto et al., 2015; Teixeira et al., 2016; Liu et al., 2020), as well as PARK10 (USP24), which is related to cancer-associated acetylation (Wang et al., 2017). In general, the significance of Parkinson family genes in cancer remains to be further explored and could be of major relevance for our understanding of cancer progression.

We have focused on exploring the role of Parkinson family genes in the occurrence and development of cancer. Our previous studies showed that PARK6 (PINK1) can promote migration and proliferation of lung cancer cells by regulating autophagy ( $\mathrm{Lu}$ et al., 2020), and PARK7 (DJ-1) is necessary for the transcription of HIF-1 $\alpha$ and survival of colorectal cancer cells (Lin et al., 2018;
Zheng et al., 2018). However, there has been no relevant research on the overall prognostic significance of Parkinson family genes in cancer. Herein, we provide a preliminary analysis of Parkinson family genes in the prognosis and treatment of lung adenocarcinoma (LUAD). The current findings will help us further understand the role of Parkinson family genes in cancer, aid in LUAD prognosis and treatment, and indicate possible directions for future research on this gene family.

\section{MATERIALS AND METHODS}

\section{Differential Gene Expression Analysis}

We downloaded the normalised gene expression data of cancer and normal tissues from the Cancer Genome Atlas (TCGA) and Genotype-Tissue Expression Project (GTEx) databases in UCSC Xena (http://xena.ucsc.edu/). Data was graphically displayed using the ggplot2 $\mathrm{R}$ package. For differences in protein expression between cancer and normal tissues, we used the clinical proteomic tumor analysis consortium (CPTAC) analysis tool of the UALCAN database (http://ualcan.path.uab. edu/index.html), and no LUAD protein expression data were found for PARK6 (PINK1), PARK10 (ELAVL4), and PARK16 (SLC41A1). The expression of Parkinson family genes at different stages of LUAD was explored using the GEPIA2 database (http:// gepia2.cancer-pku.cn/\#index).

\section{Survival Analysis}

We obtained and downloaded the gene expression and detailed pathological data of 436 LUAD patients and 425 LUSC patients (primary solid tumour samples with detailed prognostic information and a survival time of up to 15 years were included) from the SangerBox database (http://www.sangerbox. $\mathrm{com} /$ ) to analyse the impact of Parkinson family genes on cancer prognosis. Data was graphically displayed with the help of the survival $\mathrm{R}$ package. The forest plot was constructed using GraphPad Prism 8.

\section{Prognostic Model Construction and Verification}

Based on the expression and prognostic value of 25 Parkinson family genes in 436 LUAD and 425 LUSC patient samples, we used the survival $\mathrm{R}$ package to construct a Least absolute shrinkage and selection operator (LASSO) regression model. Risk factor analysis was performed using the Hiplot online analysis platform (https://hiplot.com.cn/). A prognostic nomogram model for the 436 LUAD patients was constructed using the rms $\mathrm{R}$ package, and the bootstrap method was applied to assess consistency. To validate the model in external data sets, we selected three Gene Expression Omnibus (GEO) chips (GSE37745, GSE31210, and GSE30219) of LUAD patients with detailed prognostic information. The receiver operating characteristic (ROC) curve and time-dependent ROC curve of the working characteristics of subjects were established using $\mathrm{R}$ in order to evaluate the survival prediction accuracy of the sevengene signature for the three chips. 


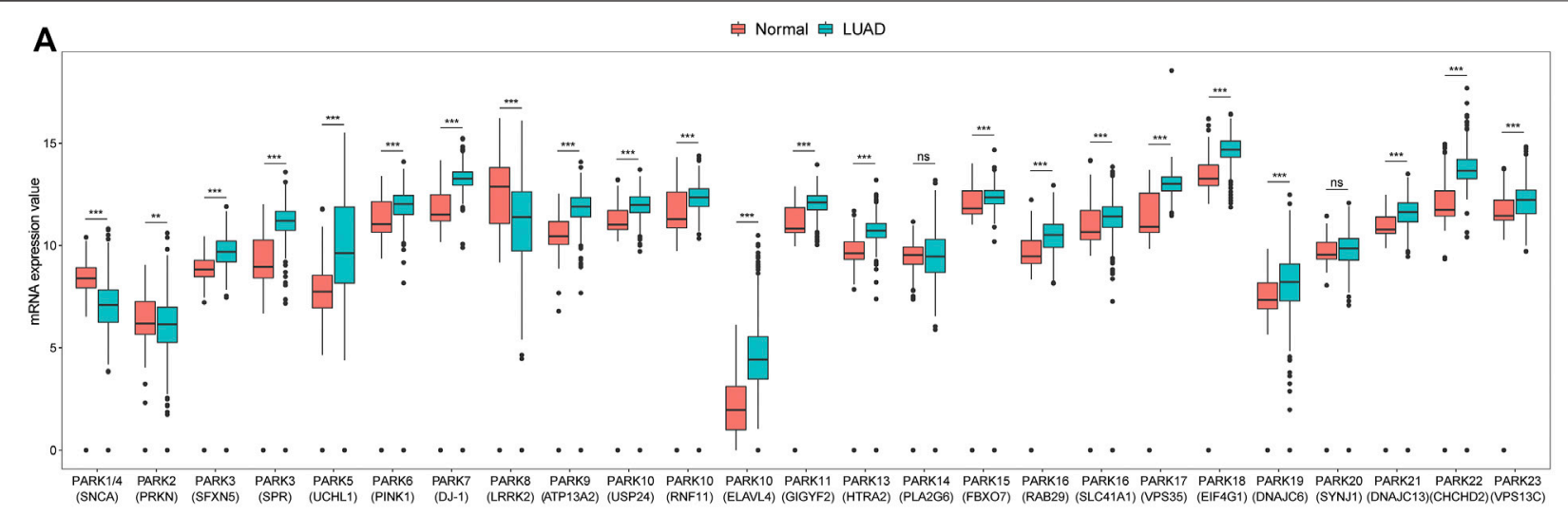

B
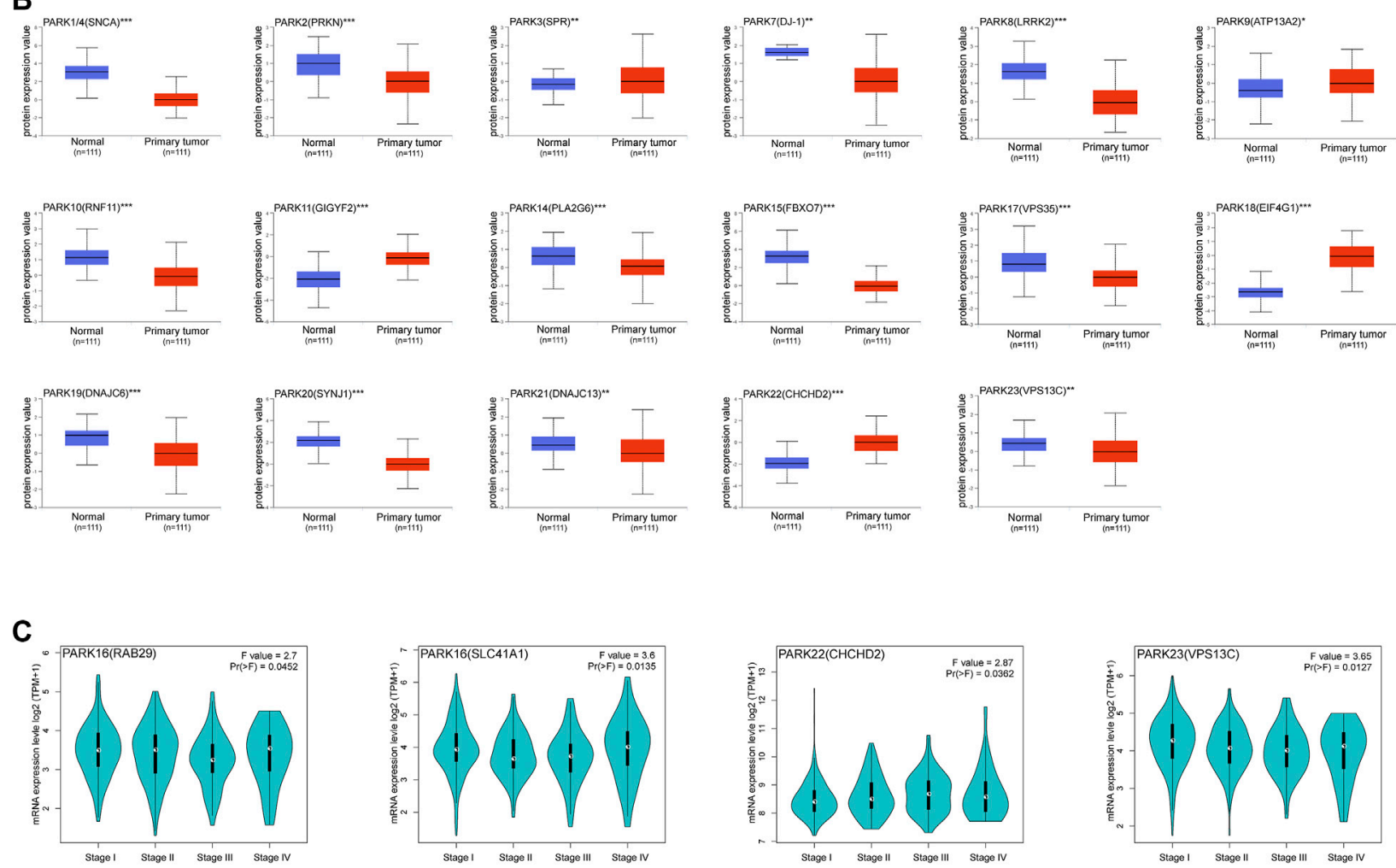

FIGURE 1 | Expression of Parkinson family genes in LUAD. (A) Differences in mRNA expression of 25 Parkinson family genes in LUAD and normal tissues from TCGA and GTEx databases (normal = 397, LUAD = 513). (B) Differences in the protein expression levels of 17 Parkinson family genes between LUAD and normal tissues in the UALCAN database. (C) Expression differences for four Parkinson family genes at different disease stages in the GEPIA2 database. ${ }^{*},{ }^{\star \star}$, and ${ }^{\star \star \star}$ represent $p<0.05$, $p<0.01$, and $p<0.001$, respectively.

\section{Bioinformatics Analysis}

Univariate and multivariate analyses were conducted using the IBM SPSS Statistics 19. The tumour mutation burden (TMB) and neoantigen (NEO) data of 436 LUAD patients were obtained from the Cancer Imaging Archive (TCIA) database (https://tcia. at/home) (Van Allen et al., 2015; Hugo et al., 2016), and both data were available only for 363 patients. We explored mutations of the seven genes screened via LASSO regression (TCGA, PanCancer Atlas) using the cBioportal database (https://www. cbioportal.org/). Employing the HitPredict database (http:// www.hitpredict.org/) (Patil and Nakamura, 2005; Patil et al., 2011; López et al., 2015), we searched for interaction partners of the seven genes. The protein network interaction map was constructed using Cytoscape_v3.7.1. GO and KEGG pathway enrichment analysis of the seven genes and Gene Set Enrichment Analysis (GSEA) analysis of high- and low-risk LUAD patients were carried out using the OmicShare 6.2.1 online tool. 


\section{Immune Cell Infiltration Analysis}

In the TIMER database (http://cistrome.dfci.harvard.edu/ TIMER/), we explored the impact of mutations in the seven genes on the degree of infiltration for six immune cell types within the tumour microenvironment (TME). Based on the ESTIMATE database (https://bioinformatics.mdanderson.org/estimate/), we further analysed the immune scores of 436 LUAD patients in different risk groups. Next, we used seven analysis methods (TIMER, CIBERSORT, CIBERSORT-ABS, QUANTISEQ, MCPCOUNTER, XCELL, and EPIC) to determine the immune cell infiltration status within the TME of patients in the TIMER2 database (http://timer.cistrome.org/). In addition, we explored differences between the high- and low-risk groups in the steps of the cancer immunity cycle for these patients using the TIP database (http://biocc.hrbmu.edu.cn/TIP/) (Xu et al., 2018).

\section{Analysis of the Efficacy and Response to Immunotherapy and Targeted Therapy}

Gene expression and detailed pathological data of anti-PD-1-treated LUAD patients were obtained from the GSE135222 dataset. The correlation between risk scores and various immunosuppressive molecules was graphically displayed utilising the online analysis tool Hiplot. R packages were used to analyse the correlation between risk score and multiple treatment targets.

\section{RESULTS}

\section{Expression of Parkinson Family Genes in LUAD}

In order to explore the expression of Parkinson family genes in LUAD, we obtained the normalised cancer and normal tissue gene expression data of LUAD patient samples from the TCGA and GTEx databases in UCSC Xena. All Parkinson family genes exhibited significant differential expression, with the exception of PARK14 (PLA2G6) and PARK20 (SYNJ1) (Figure 1A). Similar results were obtained for LUSC (Supplementary Figure S1). Furthermore, using the UALCAN database, we analysed the protein expression of Parkinson family genes between LUAD and normal tissue. Seventeen genes exhibited significant differences in expression at the protein level (Figure 1B). Finally, we explored the expression of Parkinson family genes at different TNM stages in the GEPIA2 database and found that four genes had significant expression differences during the progression of LUAD (Figure 1C). In summary, all Parkinson family genes exhibited significant differential expression in LUAD, suggestive of their involvement in the occurrence and development of lung cancer.

\section{Prognostic Value of Parkinson Family Genes in LUAD}

Next, we selected 436 LUAD patients from TCGA to investigate the impact of Parkinson family genes on the prognosis of LUAD. We found that 12 genes had significant differences based on LUAD prognosis (Figures 2A,B). Similarly, 11 genes had a significant impact on the prognosis of LUSC patients (Supplementary Figures S2A,B). Moreover, we performed GSEA analysis to explore the influence of deregulation of these genes on 50 hallmark gene sets. Results showed increased expression for these genes associated with poor prognosis can obviously activate a variety of cancer-related pathways (WNT, P53 and NOTCH pathway for LUAD; MYC, G2M checkpoint and Oxidative phosphorylation for LUSC). Interesting, whether in LUAD or LUSC, the impact of the increase of protective prognostic genes and risk genes on the 50 hallmark gene sets were obviously different and even opposed to each other, this finding may provide some help for follow-up in-depth study (Supplementary Figure S3, S4).

However, not all differentially expressed Parkinson genes (in Figure 1A) accurately predicted overall survival in LUAD patients. Therefore, we sought to create a gene signature from 25 Parkinson family genes that had the most pronounced impact on LUAD prognosis. To this end, we constructed a LASSO regression model based on the expression and prognosis data of 436 LUAD patients. We obtained a seven-gene prognostic signature (Figures 2C,D). The complete names of the seven genes and their main functions are listed in Table 1. Meanwhile, we further analyzed the correlation between the 7 genes and genes in the non-small cell lung cancer pathway (map05223) from KEGG database. The results showed that the 7 genes were related to multiple cancer progression marker genes respectively, this result suggest these genes were involved in the development of lung adenocarcinoma via different mechanisms (Supplementary Figure S5). We also constructed a LASSO regression model based on the data of LUSC patients. However, limited by data quality, the results indicated that a combination of two genes had the best performance (Supplementary Figures S2C,D). Therefore, we focused on the seven-gene signature for the prognosis and treatment outcome prediction in LUAD.

\section{Our Seven-Gene Signature can be Used as an Independent Prognostic Indicator for LUAD}

To better assess the prognostic value of our seven-gene signature in LUAD, we first conducted risk factor analysis based on the genes. The occurrence of death was significantly correlated with a higher risk factor score (Figure 3A). Furthermore, we divided 436 patients into high- and low-risk groups based on risk factor score and analysed prognosis in the two groups. The high-risk group exhibited a significantly poorer prognosis among patients with different TNM stages (Figure 3B). Univariate and multivariate analyses also indicated that the signature-based risk factor score was a superior prognostic indicator compared to TNM staging (Figures 3C,D). Taken together, the seven-gene signature of Parkinson family genes can be used as an independent prognostic marker for LUAD.

\section{Validation of the Prognostic Value of Our Seven-Gene Signature for LUAD}

In order to validate and further assess the prognostic value of our seven-gene signature for LUAD, we constructed a nomogram based on data from the TCGA database in order to predict patient 


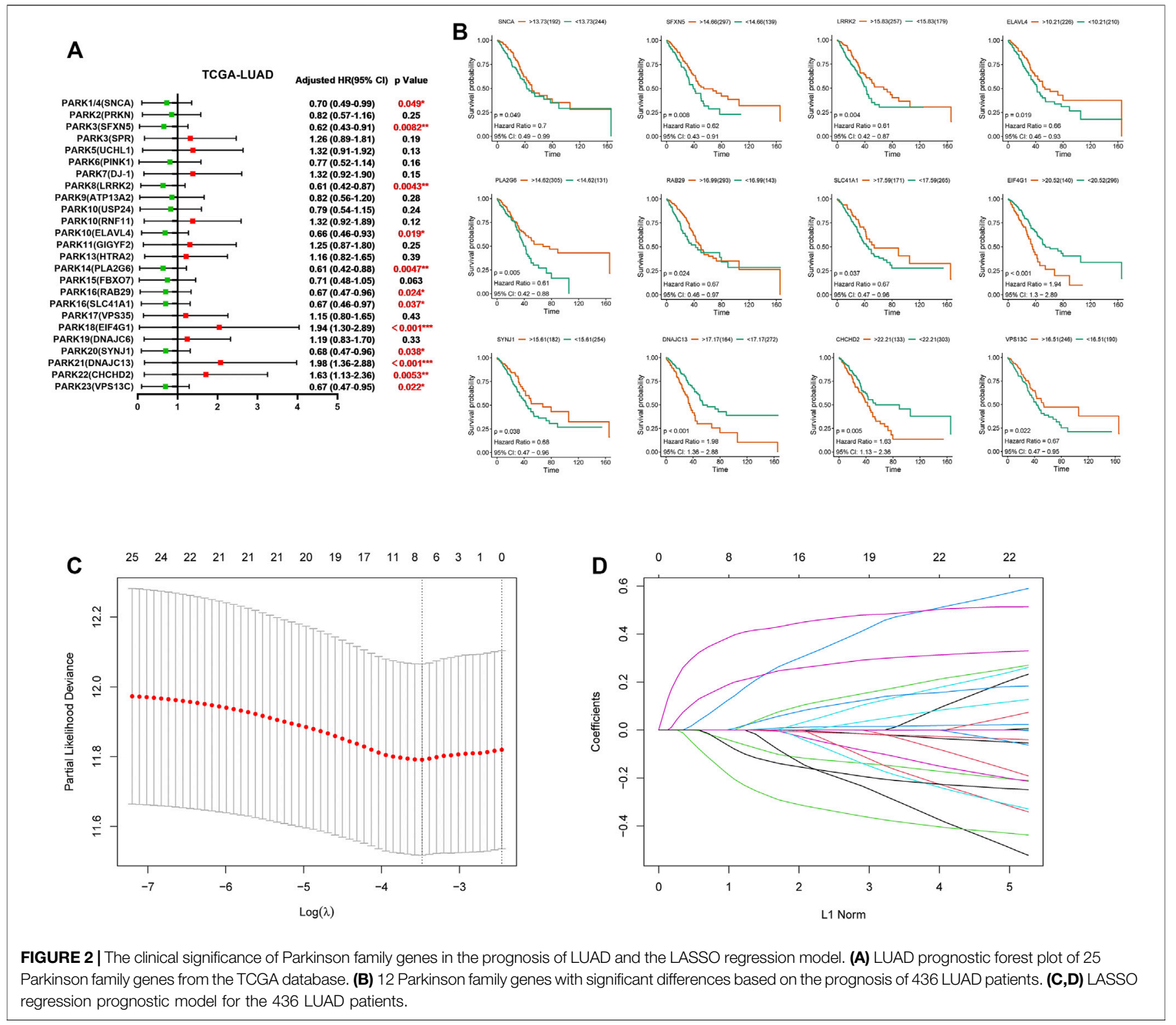

TABLE 1 | LASSO regression result of Parkinson's disease gene family in TCGA-LUAD dataset.

Gene symbol

Full name

PARK3(SFXN5)

PARK14(PLA2G6)

PARK16(RAB29)

PARK16(SLC41A1)

PARK18(EIF4G1)

PARK21(DNAJC13)

PARK22(CHCHD2) sideroflexin-5

calcium-independent phospholipase A2

ras-related protein Rab-7L1

solute carrier family 41 member 1

eukaryotic translation initiation factor 4 gamma 1

dnaJ homolog subfamily $\mathrm{C}$ member 13

coiled-coil-helix-coiled-coil-helix domain-containing

protein 2

\section{Function}

mitochondrial amino-acid transporter

involved in cellular membrane homeostasis, mitochondrial integrity and signal transduction

Involved in vesicle trafficking

a predominant Mg2+ efflux system at the plasma membrane

component of the protein complex elF4F

involved in membrane trafficking through early endosomes

transcription factor survival probability by weighing age, gender, stage, T, N, M, and the signature-based risk score (Figure 4A). Further, we applied the bootstrap method to evaluate the nomogram's predictive performance. The calibration curves indicated that the nomogram-predicted probability matched the actual 3- and 5years survival (Figures 4B,C). Subsequently, we selected three 

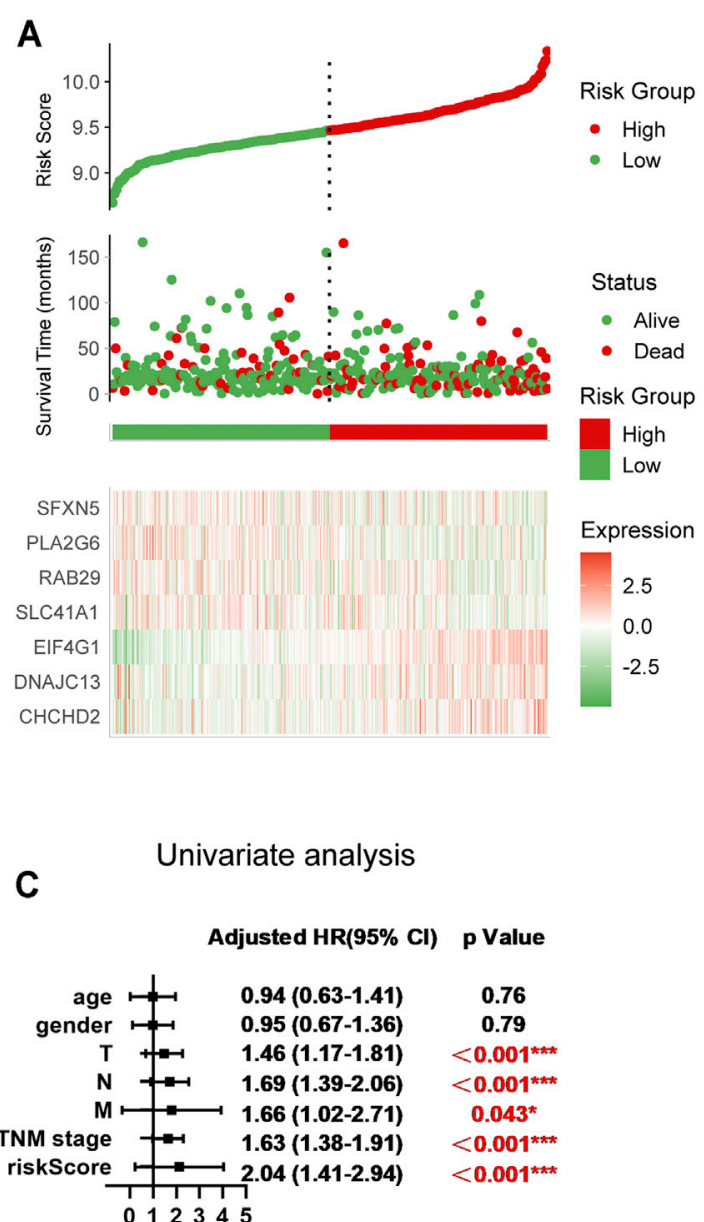
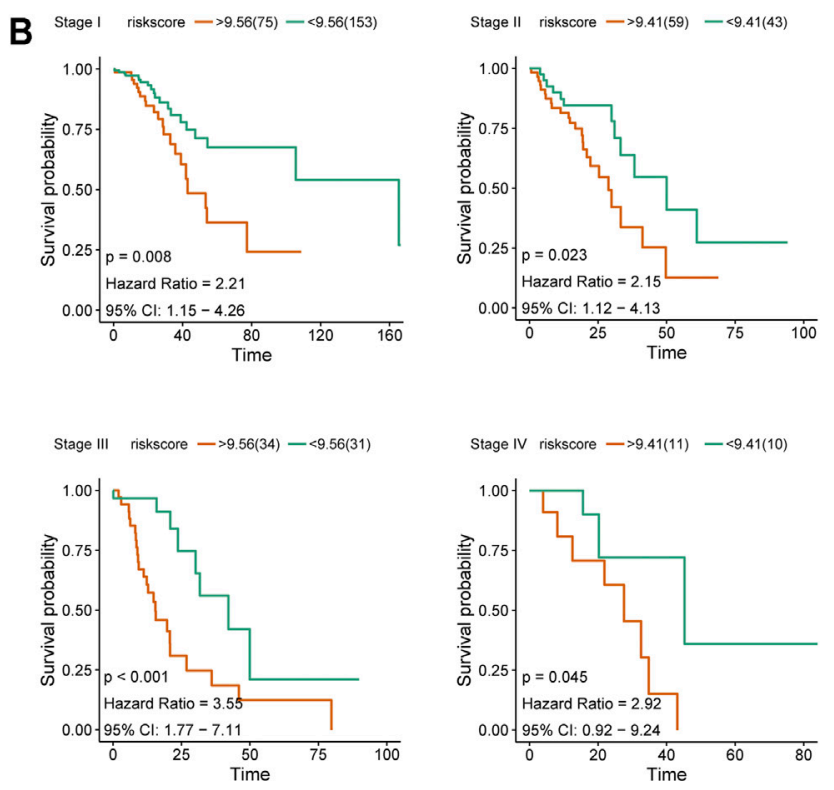

Dultivariate analysis

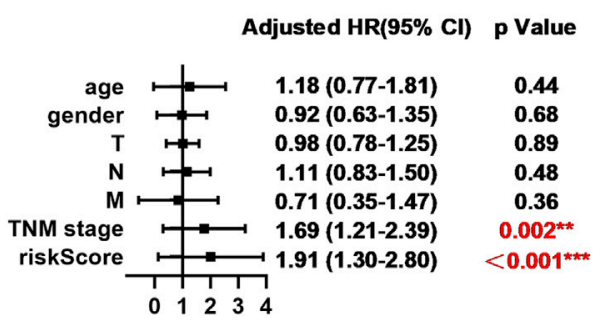

FIGURE 3 | Significance of the seven-gene prognostic model in LUAD. (A) Risk factor analysis for the 436 LUAD patients (cut-off value = 9.48). (B) Prognostic differences between high- and low-risk groups in different TNM stages in LUAD. (C,D) Univariate and multivariate analysis of the seven-gene prognostic risk model.

GEO datasets (GSE37745, GSE31210, and GSE30219) with detailed prognostic information for external testing (Table 2). The seven-gene signature risk scores suggested a significantly better diagnostic performance than TNM staging based on the ROC curve, also showing excellent prognostic performance at different survival time points in the time-dependent ROC curve (Figures 4D-F). Taken together, risk evaluation based on the seven-gene signature has important clinical significance in the diagnosis and treatment of LUAD.

\section{Functional Enrichment Analysis of the Seven Signature Genes}

We explored the possible mechanisms underlying the functional association of signature genes with poor prognosis. Utilising the HitPredict database, we searched for interaction partners of their protein products, which are displayed in the protein interaction network diagram (Figure 5A). We then performed GO and KEGG enrichment analyses for these genes. GO enrichment analysis indicated the genes' involvement in diverse tumourigenesis- and development-associated signalling, such as metabolism-related pathways, ubiquitination pathways, and RNA transcription-related processes (Figures 5B-D). Furthermore, KEGG pathway enrichment analysis also revealed that immune and cell proliferation-related pathways (RIG-I-like receptor, MAPK, mTOR, and $\mathrm{ErBb}$ ) were enriched by these genes (Figure $5 \mathrm{E}$ ).

\section{Signature Gene Expression Affects the TME in LUAD}

In view of the fact that various immune-related processes were also observed among functional enrichment results (Figure 5E), we sought to explore the influence of the seven signature genes on the TME. Employing the TIMER database, we obtained the effect of various single gene mutations on the infiltration of six immune cell types (B cells, CD8+T cells, CD4+T cells, macrophages, neutrophils, and dendritic cells) into the TME. The results indicated that mutations of signature genes decreased the degree of infiltration for almost all six immune cell types (Figures 6A-G). We also explored the link between risk factor score and TMB (or NEO) based on TCIA data. A high-risk score 


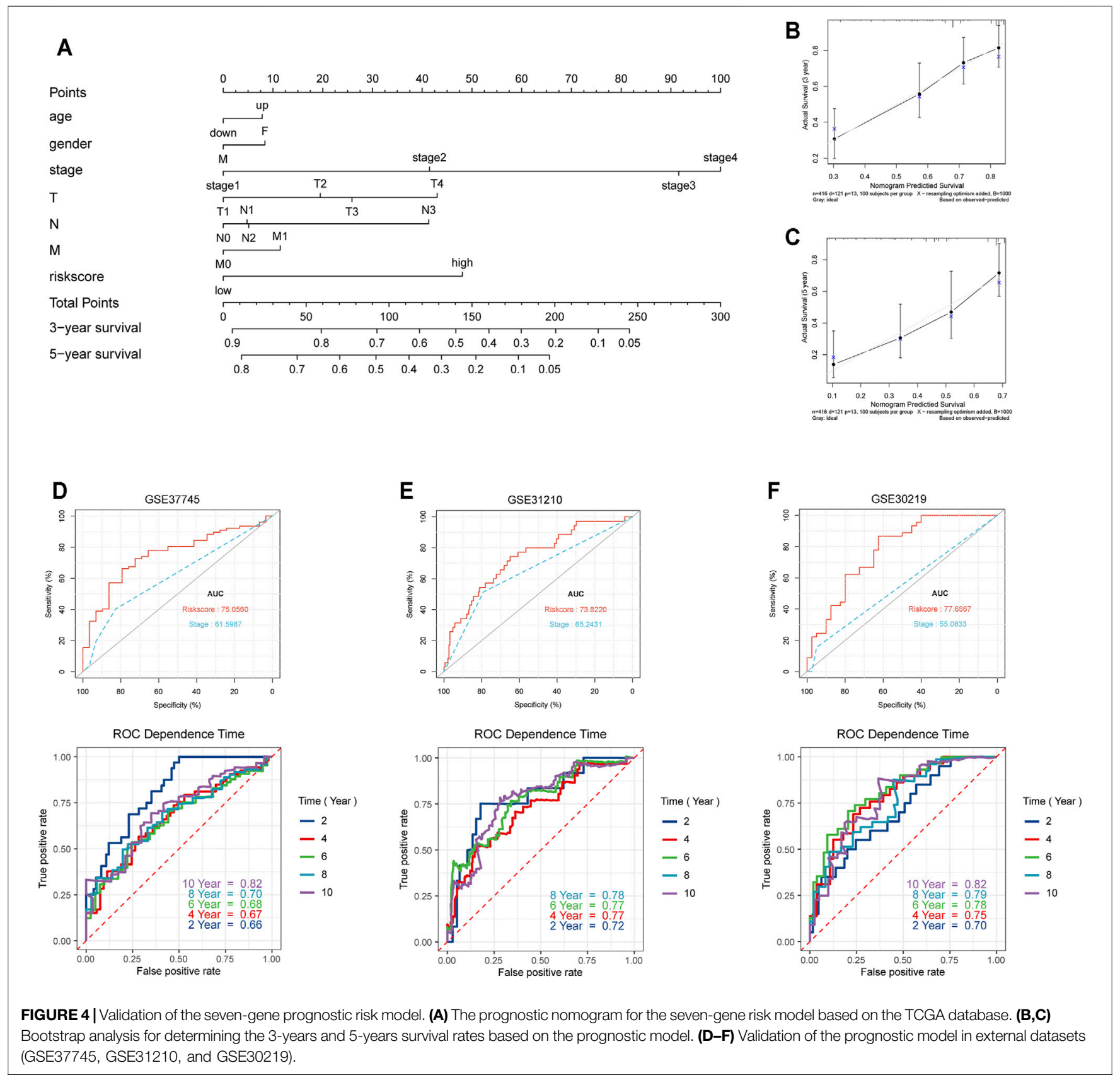

was clearly related to a high TMB and high NEO (Figure 6H). Mutation data from the cBioportal database indicated that mutations in these seven genes were rare among TCGALUAD patients (Figure 6I).

We further explored the effect of the seven-gene signature on the TME. Estimation analysis indicated that the immune score of the high-risk group was significantly lower than that of the lowrisk group for the $436 \mathrm{LUAD}$ patients (Figure 7A). We further observed the different infiltration levels of 22 immune cell types between the low- and high-risk groups using CIBERSORT (Figure 7B). Meanwhile, in order to eliminate possible errors caused by different analysis methods, we employed another six methods (Supplementary Figure S6) and summarised the results, revealing the same trend and significant differences (Table 3). However, these results revealed an interesting phenomenon. In the high-risk group, the infiltration of DC cells, B cells, CD4+ T cells, and CD8+ T cells remained lower, while M1 pro-inflammatory macrophages were upregulated, and M2 anti-inflammatory macrophages and Treg cells were downregulated.

The cancer immunity cycle reflects a complex interaction network involving the chemokine system, other immunomodulators, and the various immune cell types. Based on TIP data, we found that high risk was associated with the 
TABLE 2 | GEO dataset for the prognostic value of the 7 genes in LUAD.

\begin{tabular}{cccc}
\hline Features & GSE37745 & GSE31210 & GSE30219 \\
\hline $\begin{array}{c}\text { Platforms } \\
\text { Samples } \\
\text { adeno }\end{array}$ & GPL570 & GPL570 & GPL570 \\
else & 106 & 226 & 85 \\
Age & 90 & 20 & 222 \\
<60 & & & \\
$\geq 60$ & 64 & 105 & 118 \\
Gender & 132 & 136 & 174 \\
female & & & 43 \\
male & 89 & 130 & 250 \\
Stage & 107 & 116 & 170 \\
I & & & 59 \\
II & 130 & 168 & 55 \\
III & 35 & 58 & 8 \\
IV & 27 & 0 & \\
\hline
\end{tabular}

release of cancer cell antigens (Step 1), reduced priming and activation (Step 3), recruitment of multiple immune cells (Step 4), and the infiltration of immune cells into tumours (Step 5) (Figure 7C). It is worth noting that Th1 cells were significantly downregulated in the high-risk group, while Th2 cells were upregulated, although the difference was not statistically significant. This partly explains why the various effector cells of cellular immunity exhibited low infiltration with an opposite trend of change compared to regulatory cells. The detailed underlying mechanism remains to be further explored. Similarly, GSEA analysis showed that signaturebased high risk was associated with a significant inhibition of tumour immune-related processes (Figure 7D). Exploring the mechanism underlying the increase in M2 cells and Treg cells in the high-risk group may help explain the low infiltration of effector cells.

\section{Utility of the Seven-Gene Signature for the Prediction of Immunotherapy and Targeted Therapy Response in LUAD}

Finally, we sought to explore the utility of our seven-gene signature as a predictor of immunotherapy and targeted therapy response in LUAD. A high-risk score was significantly negatively correlated with a variety of immunosuppressive molecules (Figure 8A). Furthermore, we explored the correlation between risk score and the efficacy of PD-L1 immunotherapy in LUAD. Data from GSE135222 indicated that after PD-1 immune checkpoint inhibitor treatment, the risk score of dying patients was higher than that of surviving patients, and there was a negative correlation between the survival time for surviving patients and the risk score (Figure 8B). Limited by the sample size, the results were not statistically significant, and we did not find other available immunotherapy cohorts with detailed prognostic and gene expression data. Nevertheless, we believe that these results would be useful for treatment selection. We analysed the correlation between risk score and the expression of multiple driver genes for targeted therapy in TCGA patients, observing a clear correlation (Figure 8C). Furthermore, based on the Genomics of Drug Sensitivity in Cancer (GDSC) database, we predicted the impact of 7 genes on the half-inhibitory concentration (IC50) of some common chemotherapeutics (platinum, paclitaxel, etc) and targeted agents for non-small cell lung cancer (NSCLC). Interestingly, the result showed high expression of SLC41A1, RAB29 and PLA2G6 may be positively correlated with resistance of various common chemotherapeutics, whereas high level of CHCHD2, EIF4G1, DNAJC13 and SFXN5 may be involved in resistance of target therapy for drive gene (Supplementary Figure S7). However, we did not find available targeted therapy datasets for validation. In general, our findings indicated that targeted therapy may be more appropriate than immunotherapy for high-risk patients as a precision medicine approach for LUAD.

\section{DISCUSSION}

Lung cancer remains the major contributor to the cancer disease burden worldwide, ranking first in cancer-associated mortality. The biggest reason for this is that lung cancer patients have already entered the middle or late stages of disease upon diagnosis. Advanced disease has a strong tendency for metastasis and relapse. Although progress has been made in diagnosis and treatment strategies over the past decades, patient prognosis remains very poor, with a 5-years survival rate of only about $20 \%$ (Siegel et al., 2021). As LUAD is the major histologic subtype of lung cancer, it is necessary to explore more effective and sensitive biomarkers for its prognosis and treatment.

Instead of basing LUAD prognosis on a single gene, we screened 25 Parkinson family genes and established a sevengene prognostic signature (PARK3 [SFXN5], PARK14 [PLA2G6], PARK16 [RAB29], PARK16 [SLC41A1], PARK18 [EIF4G1], PARK21 [DNAJC13], PARK22 [CHCHD2]) for LUAD. Whether in the TCGA-LUAD cohort or the three GEO cohorts for external verification, the seven-gene signature exhibited excellent performance for LUAD prognosis. Although previous studies have shown that the signature genes were related to a variety of cancers (liver cancer, breast cancer, oral cancer, and others), there have been few studies on the mechanism through which they participate in cancer progression (Li et al., 2017; Khowal et al., 2018; Uddin et al., 2018; Yao et al., 2019). Migration, proliferation, energy metabolism, and autophagy are all considered regulatory targets, but the underlying mechanism remains to be further elucidated (Hosgood et al., 2008; Wei et al., 2015; Jaiswal et al., 2018; Ma et al., 2020; Wang et al., 2021). In addition, HIF-1a, a key regulator of the tumour hypoxic response, may be implicated in the signature genes' association with poor prognosis. Studies have shown that in non-small cell lung cancer (NSCLC), CHCHD2 and HIF-1 a co-localise in both the cytoplasm and the nucleus, with $\mathrm{CHCHD} 2$ overexpression significantly promoting that 


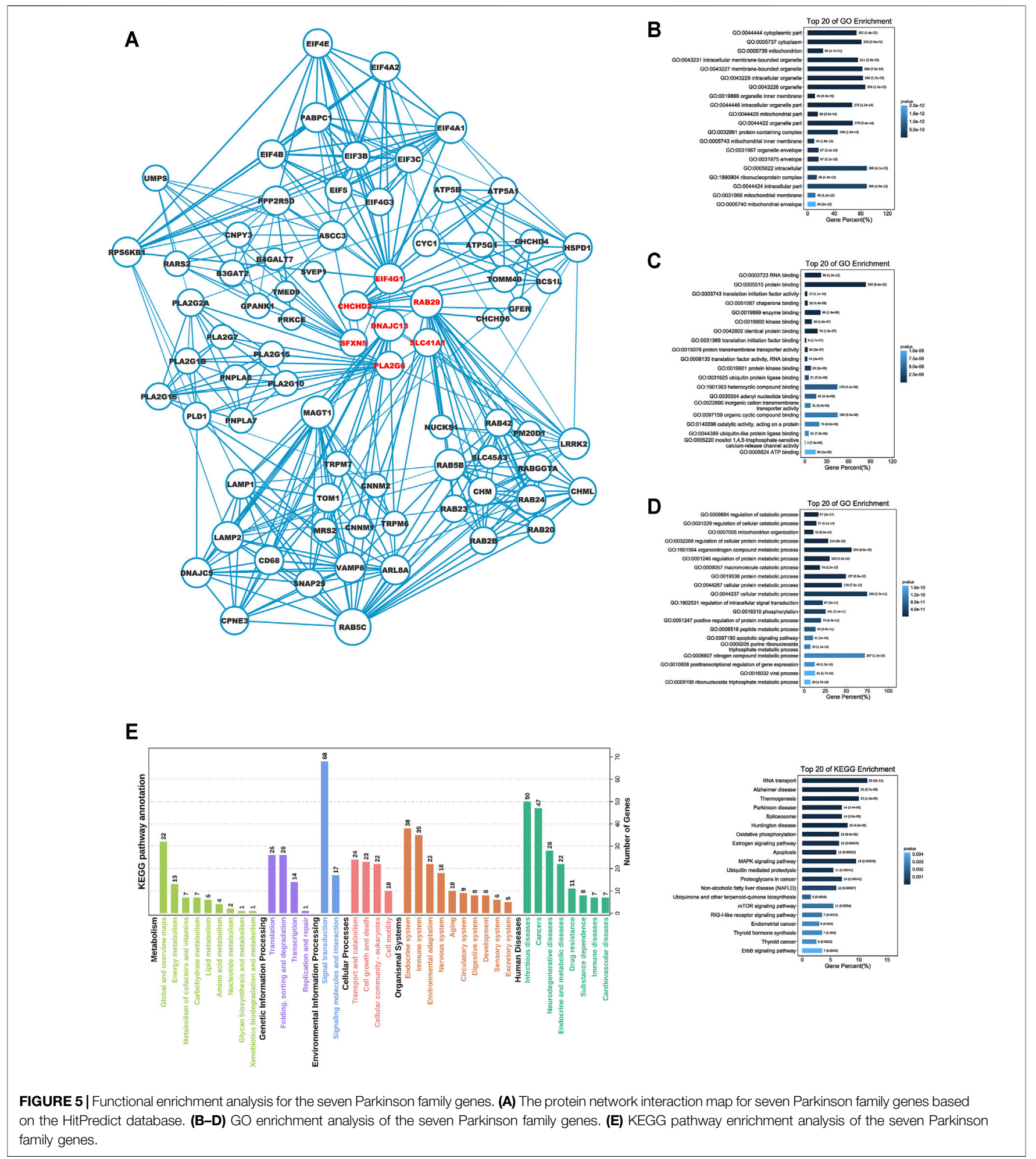

of HIF-1a (Yin et al., 2020). EIF4G1 was also confirmed as involved in HIF-1a overexpression in NSCLC (Glück et al., 2018). Furthermore, studies have suggested that EIF4G1 can also promote NSCLC progression by regulating the expression and phosphorylation of mTOR (Ser 2448) (Lu et al., 2021). In general, the current understanding of the signature genes' functions in cancer is relatively limited, necessitating further investigation.

As the seven genes belong to the Parkinson family, understanding how they contribute to PD may be conducive 

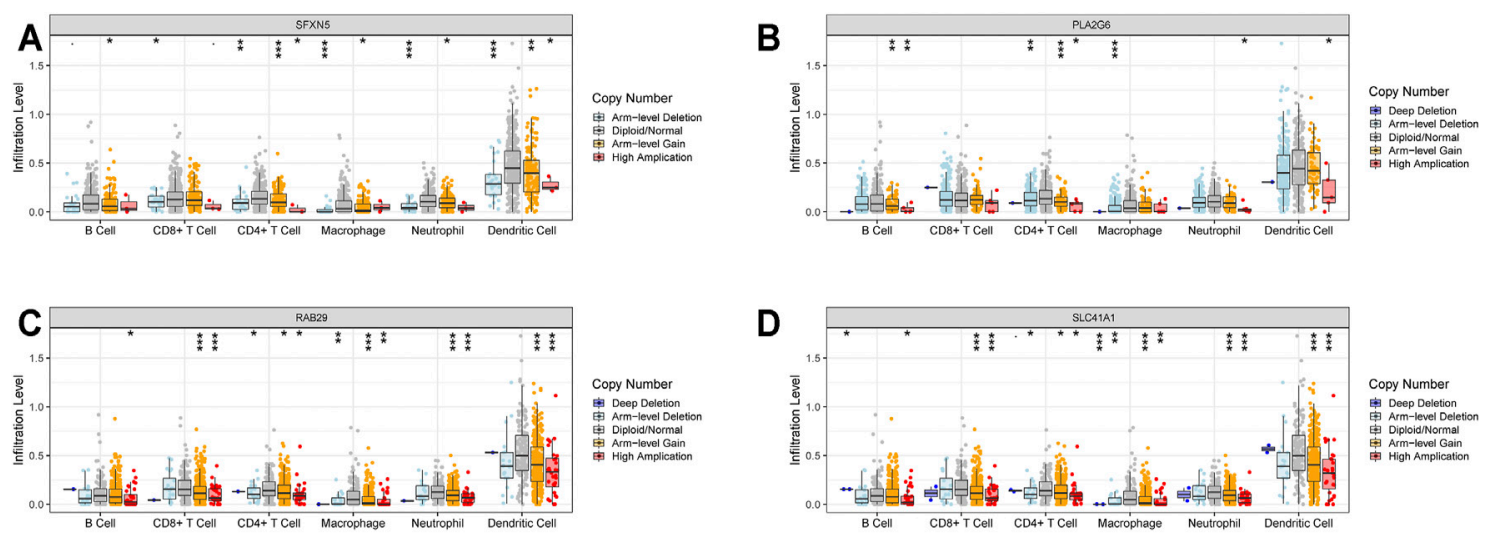

D
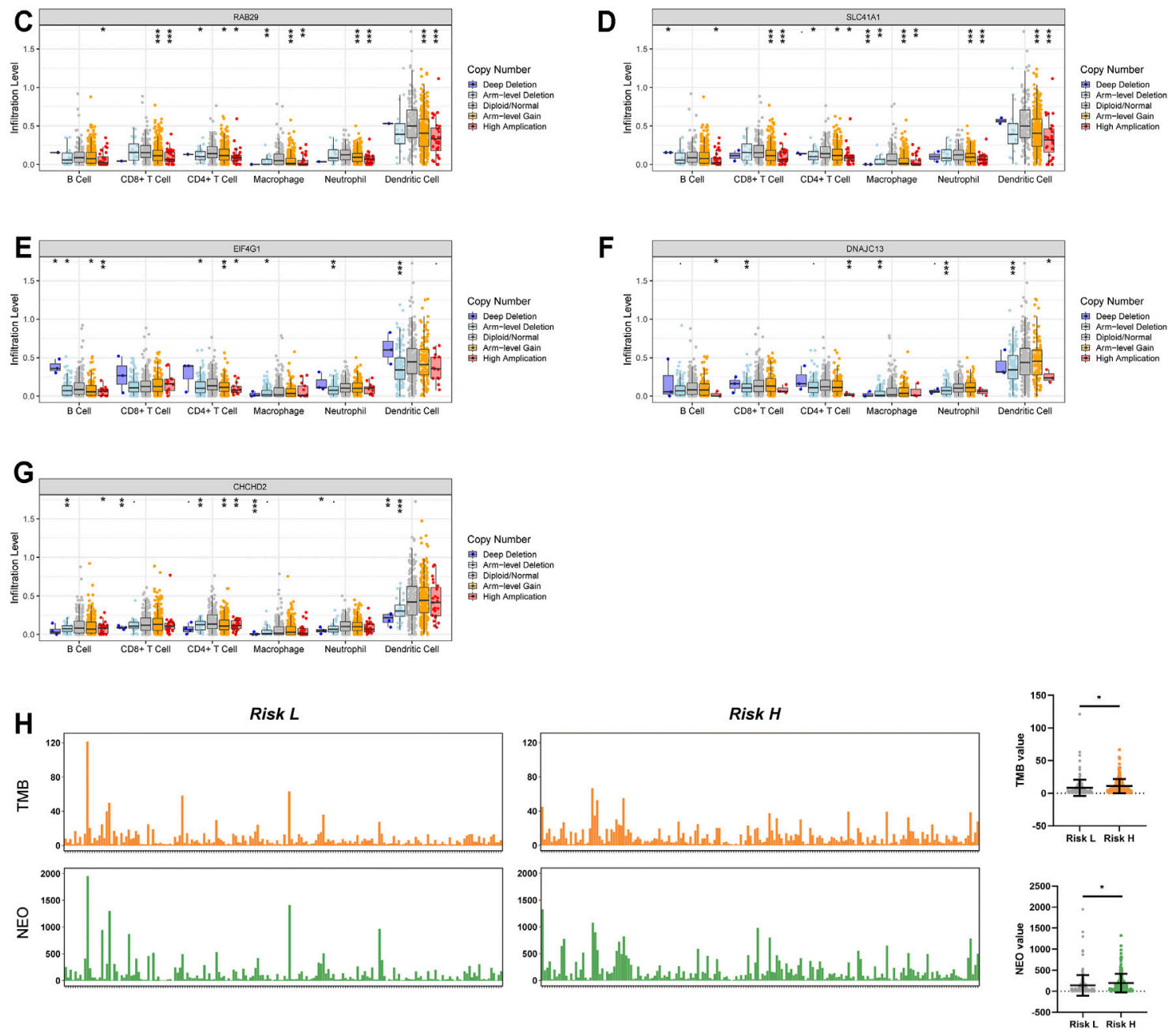

I

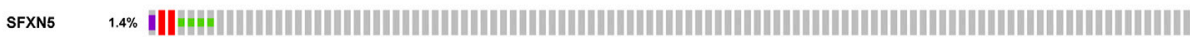

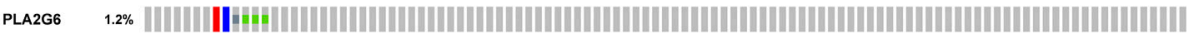 RAB29 $5 \%$.

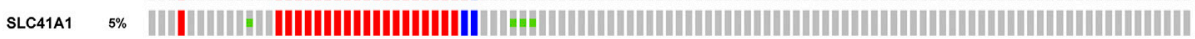

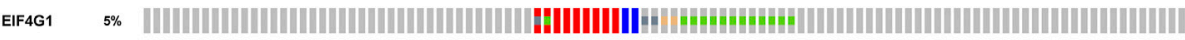

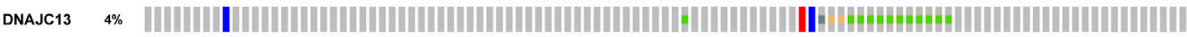

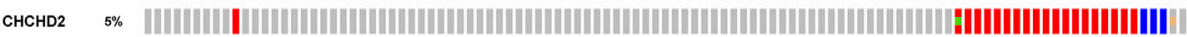

Genetic Alteration

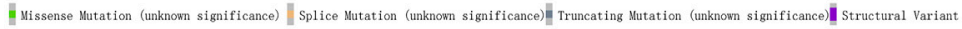

$$
\begin{aligned}
& \text { |Anplification (unknown significance Deep Deletion (unknown significance) || No alterations }
\end{aligned}
$$

FIGURE 6 | The effect of mutations in the seven Parkinson family genes on immune cell infiltration within the TME. (A-G) The effect of mutations in SFXN5, PLA2G6, RAB29, SLC41A1, EIF4G1, DNAJC13, and CHCHD2 on the infiltration of six immune cell types (B cell, CD8+T cell, CD4+T cell, macrophage, neutrophil, dendritic cell) in the TME based on the TIMER database. (H) Mutations of TMB and NEO in high- and low-risk groups in the TCGA database. (I) Mutation status of the seven Parkinson family genes in LUAD data from TCGA. 

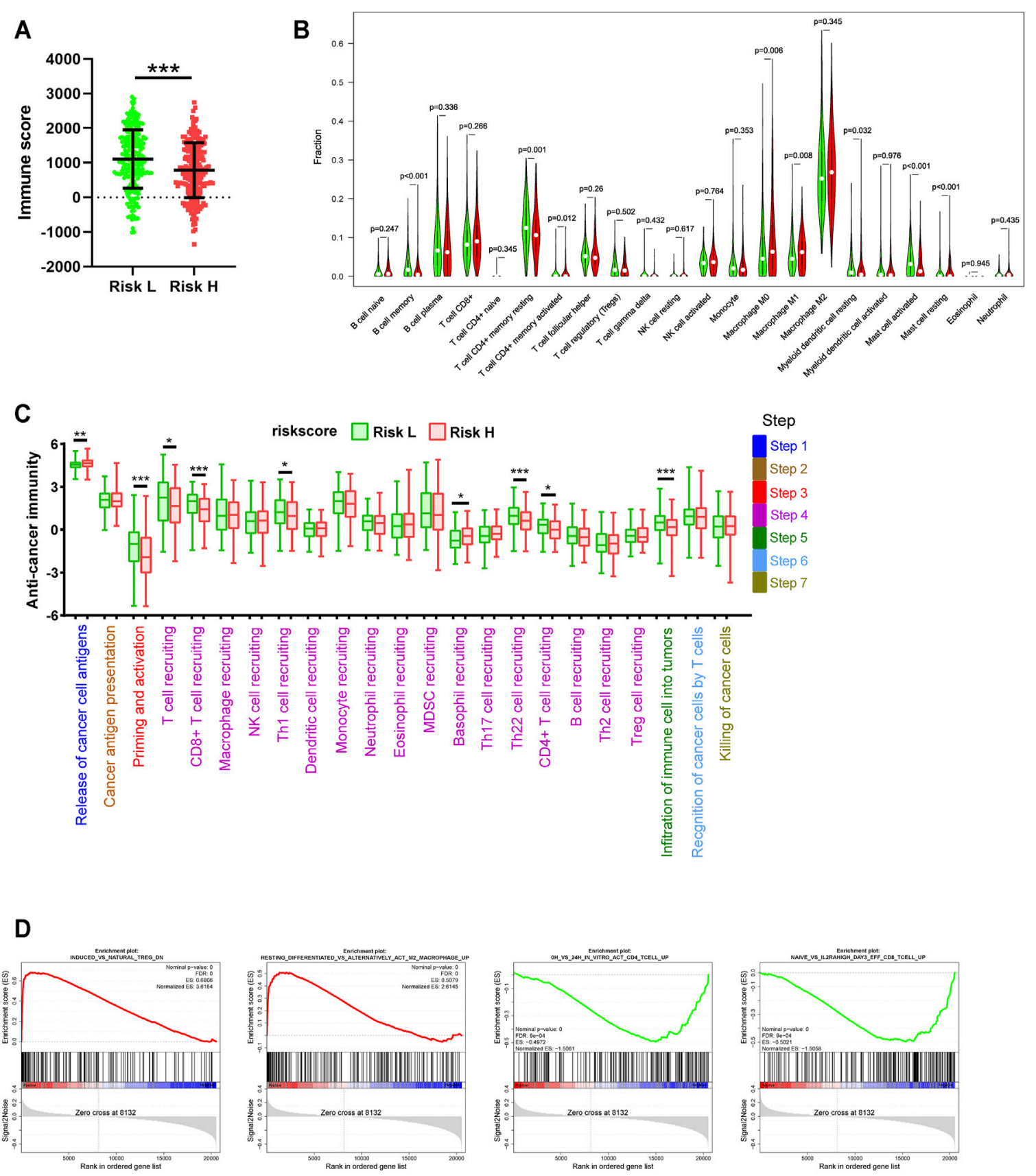

FIGURE 7 | Differences in tumor immune-related processes between high- and low-risk groups. (A) Estimation analysis of the immune scores for high- and low-risk groups. (B) Differences in the infiltration status of 22 immune cell types between high- and low-risk groups via the CIBERSORT method. (C) Differences in the cancer immunity cycle between high- and low-risk groups. (D) GSEA analysis for high- and low-risk groups based on TCGA data.

for future studies in cancer. Unfortunately, the research on SFXN5, SCL41A1, EIF4G1, and DNAJC13 in PD is limited (Deng et al., 2015; Wang et al., 2015; Puschmann, 2017), and their specific mechanisms in PD pathogenesis remain unclear. Nevertheless, some studies have provided valuable insights. PLA2G6 is believed to induce PD mainly by disturbing lipid metabolism in neurons. When lipid metabolism is dysregulated, the transient interaction between $\alpha$-synuclein and the synaptic vesicle membrane composed of phospholipids and other lipids was affected, resulting in a-synuclein aggregation and the resultant pathological a-synuclein conversion (Mori et al., 2020). At the same time, oxidative stress and inflammation caused by an imbalance of lipid metabolism also contribute to the occurrence and development of PD (Alecu and Bennett, 2019). In addition, RAB29 is believed to cause PD via 
TABLE 3 | The correlation between the risk score and immune cell infiltration level.

\begin{tabular}{|c|c|c|c|c|c|c|}
\hline & TIMER & CIBERSORT.ABS & QUANTISEQ & MCPCOUNTER & XCELL & EPIC \\
\hline B_cell & $-0.29^{\star \star \star}$ & $\begin{array}{c}\text { Naïve: }-0.06 \text {, Memory: }-0.23^{\star \star \star} \text {, } \\
\text { Plasma: }-0.14^{\star \star}\end{array}$ & $-0.25^{\star \star \star}$ & $-0.24^{\star \star \star}$ & $\begin{array}{c}\text { Naïve: }-0.12^{\star}, \text { Memory: }-0.22^{\star \star \star}, \\
\text { Plasma: }-0.14^{\star \star}\end{array}$ & $-0.23^{\star \star \star}$ \\
\hline CD4+T_cell & $-0.27^{\star \star \star}$ & $\begin{array}{l}\text { Naïve: }-0.0018, \text { Resting_memory: } \\
-0.27^{\star \star \star} \text {, Activatied_memory: } 0.11^{\star}\end{array}$ & 0.052 & Null & $\begin{aligned} \text { Naïve: } & -0.25^{\star \star \star}, \text { Non_regulatory: }-0.14^{\star \star}, \\
& \text { Central_memory: }-0.27^{\star \star \star}, \\
& \text { Effector_memory: }-0.21^{\star \star \star}\end{aligned}$ & -0.075 \\
\hline CD8+T_cell & -0.041 & -0.070 & -0.057 & -0.064 & $\begin{array}{c}\text { Naive: } 0.0055 \text {, Centra memory: }-0.16^{\star \star \star} \text {, } \\
\text { Effector memory: }-0.052\end{array}$ & $-0.15^{\star \star}$ \\
\hline Macrophage & $-0.14^{\star \star}$ & M0: $0.077, \mathrm{M} 1: 0.027^{\star}, \mathrm{M} 2:-0.17^{\star \star \star}$ & $\begin{array}{l}\text { M1: } 0.080 \\
\text { M2: }-0.31^{\star \star \star}\end{array}$ & 0.064 & $\mathrm{M} 1: 0.020^{\star}, \mathrm{M} 2:-0.26^{\star \star \star}$ & $-0.15^{\star \star}$ \\
\hline Dendritic_cell & $-0.12^{\star}$ & Resting: $-0.18^{\star \star \star}$, Activated: -0.036 & $0.12^{\star \star}$ & $-0.26^{\star \star \star}$ & Activated: $-0.18^{\star \star \star}$, Plasmacytoid: -0.051 & Null \\
\hline Tregs_cell & Null & $-0.15^{\star \star}$ & $-0.22^{\star \star \star}$ & Null & -0.037 & Null \\
\hline Mast_cell & Null & $\begin{array}{c}\text { Resting: } 0.14^{\star \star} \\
\text { Activated: }-0.31^{\star \star \star}\end{array}$ & Null & Null & $-0.17^{\star \star \star}$ & Null \\
\hline Eosinophil & Null & -0.069 & Null & Null & $-0.29^{\star \star \star}$ & Null \\
\hline Endothelial_cell & Null & Null & Null & $-0.20^{\star \star \star}$ & $-0.16^{\star \star}$ & $-0.18^{\star \star \star}$ \\
\hline
\end{tabular}

${ }^{*},{ }^{* *}$, and ${ }^{* * *}$ represent $p<0.05, p<0.01, p<0.001$, respectively.

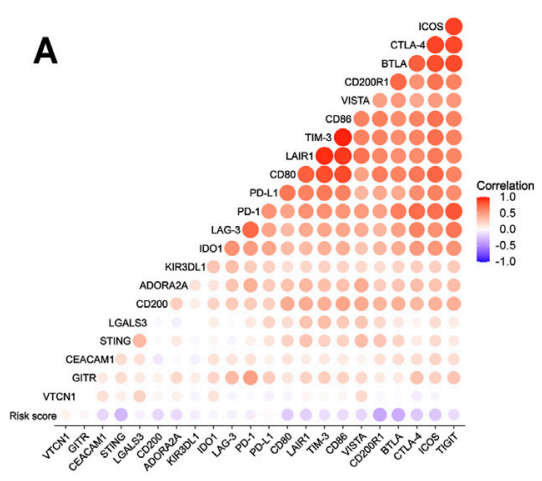

\section{B}

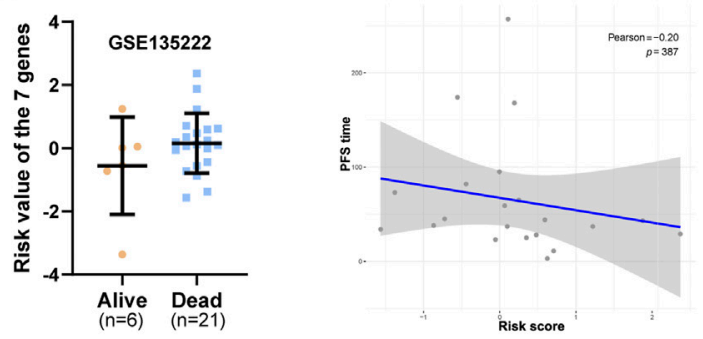

C
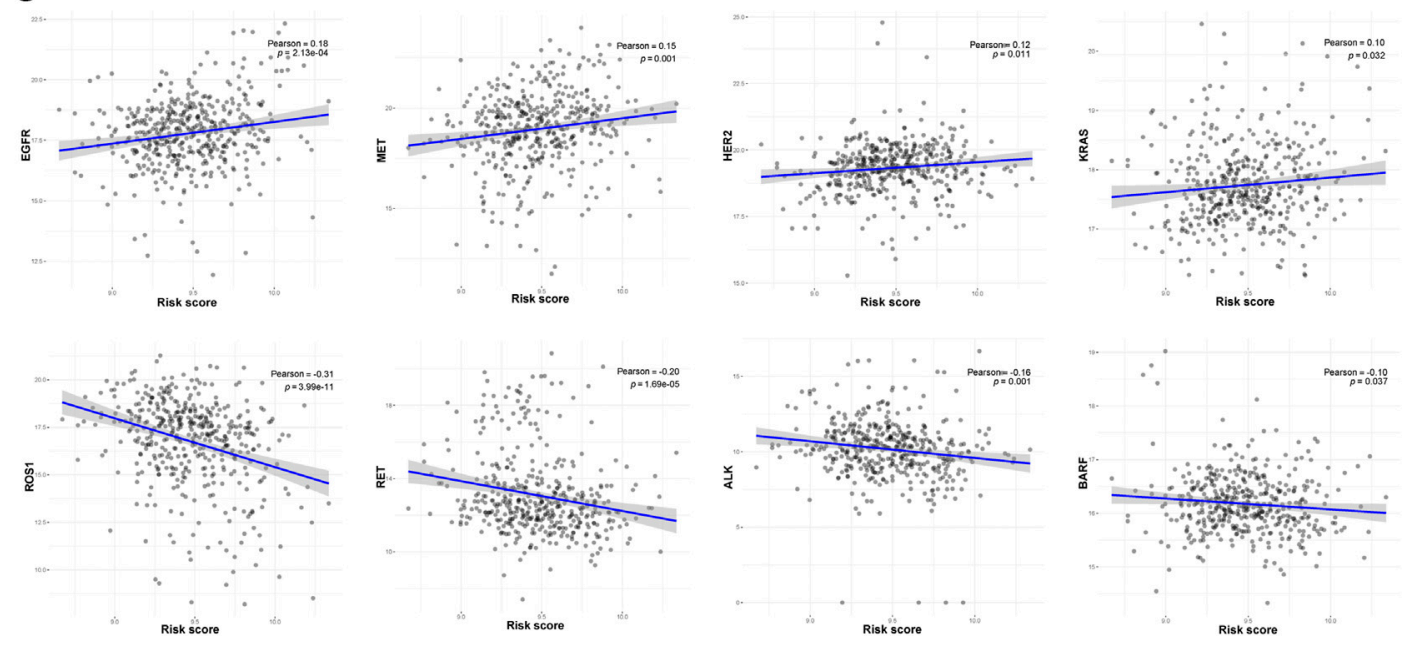

FIGURE 8 | Predictive value of the seven-gene signature for LUAD immunotherapy and targeted therapy outcomes. (A) Heat map of the correlation between risk score and the expression of multiple immunosuppressive regulatory molecules. (B) The correlation between the risk score and the efficacy of PD-1 immunotherapy based on the treatment data of GSE135222. (C) Correlation between risk score and treatment outcome of LUAD patients.

lysosomal dysfunction, while CHCHD2 may cause PD by impairing mitochondrial function (Zhou et al., 2019; Mazza et al., 2021). These processes are also of major relevance in tumours, providing an avenue for their further investigation in cancer.
Finally, we analysed the predictive merit of our seven-gene signature for LUAD treatment outcome. We observed that a high risk based on the signature may be related to low immune cell infiltration in LUAD, which was not underpinned by the polarisation of M2 macrophages and 
Treg cell infiltration. We further found that PD-1 immunotherapy may not be optimal for high-risk patients, as a high risk was related to low immune scores, suggesting targeted therapy as a more suitable option. Recent research has shown that tumour-associated macrophages (TAMs) are critical mediators of the PD-1/PD-L1 axis, and the high infiltration of M2 macrophages is significantly correlated with high PD-L1 expression within the TME (Zhu et al., 2020). Furthermore, SPP1 can promote the polarisation of M2 macrophages, and knockdown of SPP1 significantly suppressed PD-L1 expression on LUAD cells (Zhang et al., 2017). Other specific immunoregulatory mechanisms of signature genes required further investigation. Nevertheless, we consider the current findings to be of considerable value for the treatment of high-risk LUAD patients.

In summary, our study is the first to explore the role of Parkinson family genes in the prognosis and treatment of LUAD. Our research showed that the combination of seven Parkinson family genes may help predict LUAD prognosis as well as the treatment outcome for high-risk patients. Our study provides novel insight into the significance of Parkinson family genes in LUAD. We hope that the current findings will be of value for the clinical diagnosis and treatment of LUAD, and, more importantly, will further the exploration of Parkinson family genes in cancer. However, our results need more support and validation from clinical and animal models, continue to explore the unknown function and specific mechanisms of Parkinson family genes in tumors is a potential direction.

\section{REFERENCES}

Alecu, I., and Bennett, S. A. L. (2019). Dysregulated Lipid Metabolism and its Role in a-Synucleinopathy in Parkinson's Disease. Front. Neurosci. 13, 328. doi:10.3389/fnins.2019.00328

Cao, J., Chen, X., Jiang, L., Lu, B., Yuan, M., Zhu, D., et al. (2020). DJ-1 Suppresses Ferroptosis through Preserving the Activity of S-Adenosyl Homocysteine Hydrolase. Nat. Commun. 11, 1251. doi:10.1038/s41467-020-15109-y

Catalá-López, F., Suárez-Pinilla, M., Suárez-Pinilla, P., Valderas, J. M., GómezBeneyto, M., Martinez, S., et al. (2014). Inverse and Direct Cancer Comorbidity in People with Central Nervous System Disorders: A Meta-Analysis of Cancer Incidence in 577,013 Participants of 50 Observational Studies. Psychother Psychosom 83, 89-105. doi:10.1159/000356498

Chen, Y., Chen, H.-N., Wang, K., Zhang, L., Huang, Z., Liu, J., et al. (2019). Ketoconazole Exacerbates Mitophagy to Induce Apoptosis by Downregulating Cyclooxygenase-2 in Hepatocellular Carcinoma. J. Hepatol. 70, 66-77. doi:10.1016/j.jhep.2018.09.022

Deng, H., Wu, Y., and Jankovic, J. (2015). TheEIF4G1gene and Parkinson's Disease. Acta Neurol. Scand. 132, 73-78. doi:10.1111/ane.12397

Filippou, P. S., and Outeiro, T. F. (2021). Cancer and Parkinson's Disease: Common Targets, Emerging Hopes. Mov Disord. 36, 340-346. doi:10.1002/ mds. 28425

Georgakopoulos, N. D., Wells, G., and Campanella, M. (2017). The Pharmacological Regulation of Cellular Mitophagy. Nat. Chem. Biol. 13, 136-146. doi:10.1038/nchembio.2287

Glück, A. A., Orlando, E., Leiser, D., Poliaková, M., Nisa, L., Quintin, A., et al. (2018). Identification of a MET-eIF4G1 Translational Regulation axis that Controls HIF-1 $\alpha$ Levels under Hypoxia. Oncogene 37, 4181-4196. doi:10.1038/ s41388-018-0256-6

\section{DATA AVAILABILITY STATEMENT}

The original contributions presented in the study are included in the article/Supplementary Material, further inquiries can be directed to the corresponding authors.

\section{AUTHOR CONTRIBUTIONS}

JD and HZ contributed substantially to the conception and design, and gave final approval of the version to be published. $\mathrm{YL}, \mathrm{QL}, \mathrm{XL}, \mathrm{JZ}, \mathrm{ML}$, and GY contributed to the analysis and interpretation of the data. YL, HZ, and JD drafted the manuscript. $\mathrm{XD}, \mathrm{QC}, \mathrm{DZ}$, and YQ critically revised the manuscript. All authors read and approved the final manuscript.

\section{FUNDING}

This work was supported by the grants from the National Natural Science Foundation of China (81972190), Natural Science Foundation of Chongqing (cstc2018jcyjAX0205), and the Miaopu Talent Grant from Army Medical University (2019R057).

\section{SUPPLEMENTARY MATERIAL}

The Supplementary Material for this article can be found online at: https://www.frontiersin.org/articles/10.3389/fmolb.2021.735263/ full\#supplementary-material

Goto, Y., Zeng, L., Yeom, C. J., Zhu, Y., Morinibu, A., Shinomiya, K., et al. (2015). UCHL1 Provides Diagnostic and Antimetastatic Strategies Due to its Deubiquitinating Effect on HIF-1a. Nat. Commun. 6, 6153. doi:10.1038/ ncomms7153

Hosgood, H. D., Menashe, I., Shen, M., Yeager, M., Yuenger, J., Rajaraman, P., et al. (2008). Pathway-based Evaluation of 380 Candidate Genes and Lung Cancer Susceptibility Suggests the Importance of the Cell Cycle Pathway. Carcinogenesis 29, 1938-1943. doi:10.1093/carcin/bgn178

Hugo, W., Zaretsky, J. M., Sun, L., Song, C., Moreno, B. H., Hu-Lieskovan, S., et al. (2016). Genomic and Transcriptomic Features of Response to Anti-PD-1 Therapy in Metastatic Melanoma. Cell 165, 35-44. doi:10.1016/j.cell.2016.02.065

Ibáñez, K., Boullosa, C., Tabarés-Seisdedos, R., Baudot, A., and Valencia, A. (2014). Molecular Evidence for the Inverse Comorbidity between central Nervous System Disorders and Cancers Detected by Transcriptomic Meta-Analyses. Plos Genet. 10, e1004173. doi:10.1371/journal.pgen.1004173

Jaiswal, P. K., Koul, S., Shanmugam, P. S. T., and Koul, H. K. (2018). Eukaryotic Translation Initiation Factor 4 Gamma 1 (eIF4G1) Is Upregulated during Prostate Cancer Progression and Modulates Cell Growth and Metastasis. Sci. Rep. 8, 7459. doi:10.1038/s41598-018-25798-7

Jankovic, J. (2008). Parkinson's Disease: Clinical Features and Diagnosis. J. Neurol. Neurosurg. Psychiatry 79, 368-376. doi:10.1136/jnnp.2007.131045

Kang, R., Xie, Y., Zeh, H. J., Klionsky, D. J., and Tang, D. (2019). Mitochondrial Quality Control Mediated by PINK1 and PRKN: Links to Iron Metabolism and Tumor Immunity. Autophagy 15, 172-173. doi:10.1080/15548627.2018.1526611

Khowal, S., Naqvi, S. H., Monga, S., Jain, S. K., and Wajid, S. (2018). Assessment of Cellular and Serum Proteome from Tongue Squamous Cell Carcinoma Patient Lacking Addictive Proclivities for Tobacco, Betel Nut, and Alcohol: Case Study. J. Cel. Biochem. 119, 5186-5221. doi:10.1002/jcb.26554

Li, M., Li, C., Liu, W.-X., Liu, C., Cui, J., Li, Q., et al. (2017). Dysfunction of PLA2G6 and CYP2C44-Associated Network Signals Imminent Carcinogenesis from 
Chronic Inflammation to Hepatocellular Carcinoma. J. Mol. Cel Biol 9, 489-503. doi:10.1093/jmcb/mjx021

Lin, M. T., and Beal, M. F. (2006). Mitochondrial Dysfunction and Oxidative Stress in Neurodegenerative Diseases. Nature 443, 787-795. doi:10.1038/nature05292

Lin, Y., Chen, Q., Liu, Q.-X., Zhou, D., Lu, X., Deng, X.-f., et al. (2018). High Expression of DJ-1 Promotes Growth and Invasion via the PTEN-AKT Pathway and Predicts a Poor Prognosis in Colorectal Cancer. Cancer Med. 7, 809-819. doi:10.1002/cam4.1325

Liu, S., González-Prieto, R., Zhang, M., Geurink, P. P., Kooij, R., Iyengar, P. V., et al. (2020). Deubiquitinase Activity Profiling Identifies UCHL1 as a Candidate Oncoprotein that Promotes TGF $\beta$-Induced Breast Cancer Metastasis. Clin. Cancer Res. 26, 1460-1473. doi:10.1158/1078-0432.CCR-19-1373

López, Y., Nakai, K., and Patil, A. (2015). HitPredict Version 4: Comprehensive Reliability Scoring of Physical Protein-Protein Interactions from More Than 100 Species. Database 2015, bav117. doi:10.1093/database/bav117

Lu, W., Karuppagounder, S. S., Springer, D. A., Allen, M. D., Zheng, L., Chao, B., et al. (2014). Genetic Deficiency of the Mitochondrial Protein PGAM5 Causes a Parkinson'slike Movement Disorder. Nat. Commun. 5, 4930. doi:10.1038/ncomms5930

Lu, X., Liu, Q.-X., Zhang, J., Zhou, D., Yang, G.-X., Li, M.-Y., et al. (2020). PINK1 Overexpression Promotes Cell Migration and Proliferation via Regulation of Autophagy and Predicts a Poor Prognosis in Lung Cancer Cases. Cancer. Manag. Res. Vol. 12, 7703-7714. doi:10.2147/CMAR.S262466

Lu, Y., Yu, S., Wang, G., Ma, Z., Fu, X., Cao, Y., et al. (2021). Elevation of EIF4G1 Promotes Non-small Cell Lung Cancer Progression by Activating mTOR Signalling. J. Cel Mol Med 25, 2994-3005. doi:10.1111/jcmm.16340

Ma, L., Zheng, L. H., Zhang, D. G., and Fan, Z. M. (2020). CHCHD2 Decreases Docetaxel Sensitivity in Breast Cancer via Activating MMP2. Eur. Rev. Med. Pharmacol. Sci. 24, 6426-6433. doi:10.26355/eurrev_202006_21541

Mazza, M. C., Nguyen, V., Beilina, A., Karakoleva, E., Coyle, M., Ding, J., et al. (2021). Combined Knockout of Lrrk2 and Rab29 Does Not Result in Behavioral Abnormalities In Vivo. J. Parkinson. Dis. 11, 569-584. doi:10.3233/JPD-202172

Mori, A., Imai, Y., and Hattori, N. (2020). Lipids: Key Players that Modulate a-Synuclein Toxicity and Neurodegeneration in Parkinson's Disease. Int. J. Mol. Sci. 21, 3301. doi:10.3390/ijms 21093301

Ong, E. L., Goldacre, R., and Goldacre, M. (2014). Differential Risks of Cancer Types in People with Parkinson's Disease: A National Record-Linkage Study. Eur. J. Cancer 50, 2456-2462. doi:10.1016/j.ejca.2014.06.018

Patil, A., Nakai, K., and Nakamura, H. (2011). HitPredict: a Database of Quality Assessed Protein-Protein Interactions in Nine Species. Nucleic Acids Res. 39, D744-D749. doi:10.1093/nar/gkq897

Patil, A., and Nakamura, H. (2005). Filtering High-Throughput Protein-Protein Interaction Data Using a Combination of Genomic Features. Bmc Bioinformatics 6, 100. doi:10.1186/1471-2105-6-100

Peretz, C., Gurel, R., Rozani, V., Gurevich, T., El-Ad, B., Tsamir, J., et al. (2016). Cancer Incidence Among Parkinson's Disease Patients in a 10-yrs TimeWindow Around Disease Onset: A Large-Scale Cohort Study. Parkinsonism Relat. Disord. 28, 68-72. doi:10.1016/j.parkreldis.2016.04.028

Puschmann, A. (2017). New Genes Causing Hereditary Parkinson's Disease or Parkinsonism. Curr. Neurol. Neurosci. Rep. 17, 66. doi:10.1007/s11910-017$0780-8$

Siegel, R. L., Miller, K. D., Fuchs, H. E., and Jemal, A. (2021). Cancer Statistics, 2021. CA A. Cancer J. Clin. 71, 7-33. doi:10.3322/caac.21654

Tatsuta, T., and Langer, T. (2008). Quality Control of Mitochondria: Protection against Neurodegeneration and Ageing. Embo J. 27, 306-314. doi:10.1038/ sj.emboj.7601972

Teixeira, F. R., Randle, S. J., Patel, S. P., Mevissen, T. E. T., Zenkeviciute, G., Koide, T., et al. (2016). Gsk $3 \beta$ and Tomm 20 Are Substrates of the SCFFbxo7/PARK15 Ubiquitin Ligase Associated with Parkinson's Disease. Biochem. J. 473, 3563-3580. doi:10.1042/BCJ20160387

Uddin, M., Balaravi Pillai, B., Tha, K., Ashaie, M., Karim, M., and Chowdhury, E. (2018). Carbonate Apatite Nanoparticles-Facilitated Intracellular Delivery of siRNA(s) Targeting Calcium Ion Channels Efficiently Kills Breast Cancer Cells. Toxics 6, 34. doi:10.3390/toxics6030034

Van Allen, E. M., Miao, D., Schilling, B., Shukla, S. A., Blank, C., Zimmer, L., et al. (2015). Genomic Correlates of Response to CTLA-4 Blockade in Metastatic Melanoma. Science 350, 207-211. doi:10.1126/science.aad0095

Wang, F., Liao, Y., Zhang, M., Zhu, Y., Wang, W., Cai, H., et al. (2021). N6methyladenosine Demethyltransferase FTO-Mediated Autophagy in Malignant
Development of Oral Squamous Cell Carcinoma. Oncogene 40, 3885-3898. doi:10.1038/s41388-021-01820-7

Wang, L., Cheng, L., Li, N.-N., Yu, W.-J., Sun, X.-Y., and Peng, R. (2015). Genetic Analysis ofSLC41Alin Chinese Parkinson's Disease Patients. Am. J. Med. Genet. 168, 706-711. doi:10.1002/ajmg.b.32365

Wang, S.-A., Wang, Y.-C., Chuang, Y.-P., Huang, Y.-H., Su, W.-C., Chang, W.-C., et al. (2017). EGF-mediated Inhibition of Ubiquitin-specific Peptidase 24 Expression Has a Crucial Role in Tumorigenesis. Oncogene 36, 2930-2945. doi:10.1038/onc.2016.445

Wei, Y., Vellanki, R. N., Coyaud, É., Ignatchenko, V., Li, L., Krieger, J. R., et al. (2015). CHCHD2 Is Coamplified with EGFR in NSCLC and Regulates Mitochondrial Function and Cell Migration. Mol. Cancer Res. 13, 1119-1129. doi:10.1158/1541-7786.MCR-14-0165-T

Xie, Y., Liu, J., Kang, R., and Tang, D. (2021). Mitophagy in Pancreatic Cancer. Front. Oncol. 11, 616079. doi:10.3389/fonc.2021.616079

Xu, L., Deng, C., Pang, B., Zhang, X., Liu, W., Liao, G., et al. (2018). TIP: A Web Server for Resolving Tumor Immunophenotype Profiling. Cancer Res. 78, 6575-6580. doi:10.1158/0008-5472.CAN-18-0689

Yan, C., Gong, L., Chen, L., Xu, M., Abou-Hamdan, H., Tang, M., et al. (2020). PHB2 (Prohibitin 2) Promotes PINK1-PRKN/Parkin-dependent Mitophagy by the PARL-PGAM5-PINK1 axis. Autophagy 16, 419-434. doi:10.1080/ 15548627.2019.1628520

Yao, Y., Su, J., Zhao, L., Li, R., Liu, K., and Wang, S. (2019). CHCHD2 Promotes Hepatocellular Carcinoma and Indicates Poor Prognosis of Hepatocellular Carcinoma Patients. J. Cancer 10, 6822-6828. doi:10.7150/jca.31158

Yin, X., Xia, J., Sun, Y., and Zhang, Z. (2020). CHCHD2 Is a Potential Prognostic Factor for NSCLC and Is Associated with HIF-1a Expression. Bmc Pulm. Med. 20, 40. doi:10.1186/s12890-020-1079-0

Youle, R. J., and Narendra, D. P. (2011). Mechanisms of Mitophagy. Nat. Rev. Mol. Cel Biol 12, 9-14. doi:10.1038/nrm3028

Zacksenhaus, E., Shrestha, M., Liu, J. C., Vorobieva, I., Chung, P. E. D., Ju, Y., et al. (2017). Mitochondrial OXPHOS Induced by RB1 Deficiency in Breast Cancer: Implications for Anabolic Metabolism, Stemness, and Metastasis. Trends Cancer 3, 768-779. doi:10.1016/j.trecan.2017.09.002

Zhang, Y., Du, W., Chen, Z., and Xiang, C. (2017). Upregulation of PD-L1 by SPP1 Mediates Macrophage Polarization and Facilitates Immune Escape in Lung Adenocarcinoma. Exp. Cel Res. 359, 449-457. doi:10.1016/ j.yexcr.2017.08.028

Zheng, H., Zhou, C., Lu, X., Liu, Q., Liu, M., Chen, G., et al. (2018). DJ-1 Promotes Survival of Human colon Cancer Cells under Hypoxia by Modulating HIF-1a Expression through the PI3K-AKT Pathway. Cancer Manag. Res. 10, 4615-4629. doi:10.2147/CMAR.S172008

Zhou, W., Ma, D., Sun, A. X., Tran, H.-D., Ma, D.-1., Singh, B. K., et al. (2019). PDlinked CHCHD2 Mutations Impair CHCHD10 and MICOS Complex Leading to Mitochondria Dysfunction. Hum. Mol. Genet. 28, 1100-1116. doi:10.1093/ hmg/ddy 413

Zhu, Z., Zhang, H., Chen, B., Liu, X., Zhang, S., Zong, Z., et al. (2020). PD-L1Mediated Immunosuppression in Glioblastoma Is Associated with the Infiltration and M2-Polarization of Tumor-Associated Macrophages. Front. Immunol. 11, 588552. doi:10.3389/fimmu.2020.588552

Conflict of Interest: The authors declare that the research was conducted in the absence of any commercial or financial relationships that could be construed as a potential conflict of interest.

Publisher's Note: All claims expressed in this article are solely those of the authors and do not necessarily represent those of their affiliated organizations, or those of the publisher, the editors and the reviewers. Any product that may be evaluated in this article, or claim that may be made by its manufacturer, is not guaranteed or endorsed by the publisher.

Copyright $\odot 2021$ Li, Lu, Zhang, Liu, Zhou, Deng, Qiu, Chen, Li, Yang, Zheng and Dai. This is an open-access article distributed under the terms of the Creative Commons Attribution License (CC BY). The use, distribution or reproduction in other forums is permitted, provided the original author $(s)$ and the copyright owner $(s)$ are credited and that the original publication in this journal is cited, in accordance with accepted academic practice. No use, distribution or reproduction is permitted which does not comply with these terms. 